Check for updates

Cite this: Mater. Adv., 2020

1,1439

Received 7th June 2020

Accepted 11th July 2020

DOI: $10.1039 / \mathrm{d} 0 \mathrm{ma} 00386 \mathrm{~g}$

rsc.li/materials-advances

\section{Lysozyme coated copper nanoclusters for green fluorescence and their utility in cell imaging}

\begin{abstract}
Atul Gajanan Thawari, ${ }^{a}$ Piyush Kumar, ${ }^{\mathrm{b}}$ Rohit Srivastava (D) $^{\mathrm{b}}$ and Chebrolu Pulla Rao (D) $* a c$
Fluorescent, $\mathrm{pH}$ dependent and water soluble copper nanoclusters (CuNCs) were synthesized using lysozyme (lyz) as the stabilizing agent to give lysozyme coated copper nanoclusters, viz., lyz-CuNCs. The lyz-CuNCs were 3-5 $\mathrm{nm}$ in size at neutral $\mathrm{pH}$ and exhibit green fluorescence $\left(\lambda_{\mathrm{em}} \sim 510 \mathrm{~nm}\right)$ when excited at $490 \mathrm{~nm}$ and exhibited a maximum quantum yield of $18 \%$. However, under basic conditions, aggregates of lyz-CuNCs were seen with a particle size of $\sim 100 \mathrm{~nm}$. The emission of lyz-CuNCs observed at $510 \mathrm{~nm}$ complements that from the blue $\left(\lambda_{\mathrm{em}}=450 \mathrm{~nm}\right)$ and the red $\left(\lambda_{\mathrm{em}}=650 \mathrm{~nm}\right)$ nanoclusters and thus bridges the gap. On the other hand, under acidic conditions, these show a size of 5-10 nm but weakly fluoresce. The particle size and aggregations were monitored by TEM studies carried out using the samples prepared under both the acidic and the basic conditions. The lyz-CuNCs prepared at neutral $\mathrm{pH}$ show $>90 \%$ cell viability and hence can be used as a probe for cellular imaging. The imaging was carried out with both healthy and cancer cell lines, viz., NIH3T3 cells (mouse embryonic fibroblast cell), MCF7 cells (human breast cancer cells) and MDA-MB-231 cells (human estrogen negative breast cancer cells). The Z-stack study suggested the presence of lyz-CuNCs in the cells in cytoplasm. Thus, the green fluorescent lyz-CuNCs can be an alternate to green fluorescent protein (GFP) that is used for cell imaging purposes, since the latter needs a tedious procedure to express, purify and to conjugate.
\end{abstract}

\section{Introduction}

Metal nanoparticles of 3-5 nm show new physical and chemical properties owing to their nano-metric size ${ }^{1}$ and hence can be used in a broad range of applications, including, cell labeling, ${ }^{2}$ ion sensing, ${ }^{3}$ catalysis ${ }^{4}$ and anti-cancer activity. ${ }^{5}$ The recent literature primarily deals with the luminescent nanoclusters of $\mathrm{Au}^{6,7}$ and $\mathrm{Ag}^{8}$ stabilized by a variety of small chemical species as well as large biological molecules. To date, no abundant information is available regarding the synthesis of copper nanoparticles (CuNPs) and nanoclusters (CuNCs) due to the problems arising from the stability and the size of such nanoparticles. ${ }^{9-11}$

However, the CuNPs have recently attracted special attention because of their low cost and novel optical, electrical, mechanical and thermal properties. ${ }^{5}$ The CuNPs are used in textiles, paints, plastics and food industries ${ }^{3,4}$ due to their antimicrobial properties. The protein coated CuNPs are expected to exhibit anticancer activity owing to their redox ability. ${ }^{5}$ Recent studies showed that the fluorescent CuNPs are stabilized by proteins,

\footnotetext{
${ }^{a}$ Bioinorganic Laboratory, Department of Chemistry, Indian Institute of Technology Bombay, Powai, Mumbai - 400076, India

${ }^{b}$ Department of Bioscience and Bioengineering, Indian Institute of Technology Bombay, Powai, Mumbai - 400076, India

${ }^{c}$ Department of Chemistry, Indian Institute of Technology Tirupati, Settipalli post, Tirupati - 517 506, Andhra Pradesh, India. E-mail: cprao@iittp.ac.in;

Fax: +910877250 3004; Tel: +9108772503036
}

such as BSA, which gives red fluorescence, and lysozyme, which gives both red and blue fluorescence. ${ }^{12-18}$ Therefore, the stabilization of fluorescent nanoclusters of copper emitting in the visible region is still a challenging task. Proteins have been widely used in directing the synthesis of functional nanomaterials owing to the coordinating ability of $-\mathrm{NH}_{2},-\mathrm{COOH},-\mathrm{CONH}-,-\mathrm{OH}$ and $-\mathrm{SH}$ groups present as side chains in proteins. ${ }^{18}$ The use of lysozyme in the present study derives its impetus from (i) high $\mathrm{pH}$ stability due to the presence of six Lys and eleven $\operatorname{Arg}\left(\mathrm{p} K_{\mathrm{a}}=10\right)$ residues, (ii) coordination ability towards metal ions through carboxylates (Asp and Glu) and imidazole (His), (iii) ability to reduce metal ions such as $\mathrm{Cu}^{2+}, \mathrm{Ag}^{+}$, and $\mathrm{Au}^{+}$using Tyr side chains ${ }^{19}$ and (iv) its well-known antibacterial property. ${ }^{20}$ Hence, lysozyme has been widely used for the synthesis of metal nanoclusters, such as copper, ${ }^{13}$ gold $^{21,22}$ and silver $^{23}$ nanoclusters. Herein, we report the lysozyme (lyz) directed synthesis of stable, water-soluble and green fluorescent copper nanoclusters lyz-CuNCs, with excitation $\left(\lambda_{\mathrm{ex}}\right)$ at $490 \mathrm{~nm}$ and emission $\left(\lambda_{\mathrm{em}}\right)$ at $510 \mathrm{~nm}$ and demonstrated their utility in cell imaging.

\section{Experimental}

\section{Preparation of copper nanoclusters coated with lysozyme (lyz-CuNCs)}

Thirty $\mathrm{mg}$ of lysozyme was added to $3 \mathrm{ml}$ of water. To this $100 \mu \mathrm{l}$ of $100 \mathrm{mM} \mathrm{CuCl} 2$ was added. The solution was stirred for 
$30 \mathrm{~min}$ and to this $100 \mu \mathrm{l}$ of $1 \mathrm{M} \mathrm{NaOH}$ was added in $10 \mu \mathrm{l}$ portions each time ten times over a period of $10 \mathrm{~min}$ and stirred for another $30 \mathrm{~min}$. Then $100 \mu \mathrm{l}$ of hydrazine was similarly added over a period of $10 \mathrm{~min}$ and stirred for one day. The $\mathrm{pH}$ of the resultant solution was 12 . The $\mathrm{pH}$ of the solution was brought down to 7, 5 and 3 in different vials by adding requisite volume of dil $\mathrm{HCl}$ and the final volume was maintained at $10 \mathrm{ml}$ and the solutions were stirred for one day. At $\mathrm{pH}=7$, the lyz-CuNC solutions are stable for more than a month as confirmed by fluorescence spectral measurements. In order to remove the un-reacted small molecular components, the resultant solutions were dialyzed against water for $24 \mathrm{~h}$ by replacing the medium three times intermittently. The dialyzed samples showed similar characteristics to the un-dialyzed ones as proven by fluorescence and TEM studies. The dialyzed lyz-CuNCs were used for cell imaging studies.

\section{Quantum yield (QY) measurement}

The fluorescence quantum yields of lyz-CuNCs were determined using the equation, ${ }^{24}$

$$
\Phi \mathrm{X}=\left(\Phi_{\mathrm{ST}} \times A_{\mathrm{ST}} \times F_{\mathrm{X}} \times \eta_{\mathrm{X}}^{2}\right) /\left(A_{\mathrm{X}} \times F_{\mathrm{ST}} \times \eta_{\mathrm{ST}}^{2}\right),
$$

where $\Phi, A$ and $F$ refer to the quantum yield, absorbance and fluorescence intensity in the solvent having a refractive index of ' $\eta$ '. The subscripts ST and $\mathrm{X}$ refer to standard and unknown respectively. In the present case, $\Phi$ values were measured in water with $\eta=1.33$. The emission spectra of the lyz-CuNC samples were recorded at an excitation wavelength of $490 \mathrm{~nm}$ at $\mathrm{pH}$ values of $3,5,7,10$ and 12, and keeping the slit width at $3 \mathrm{~nm}$. The quinine hemisulfate in $0.1 \mathrm{M} \mathrm{H}_{2} \mathrm{SO}_{4}$ was used as a reference having a quantum yield of $0.546\left(\lambda_{\mathrm{ex}}=310 \&\right.$ $\left.\lambda_{\mathrm{em}}=455 \mathrm{~nm}\right)$.

\section{Sample preparation and studies}

Fluorescence studies were performed on a PerkinElmer LS55 Fluorescence Spectrometer. The lyz-CuNC solutions were used for the fluorescence studies by exciting these at $490 \mathrm{~nm}$ and measuring the spectra from 500 to $700 \mathrm{~nm}$ using a $5 \mathrm{~nm}$ slit width for the excitation and emission gates at a scan speed of $300 \mathrm{~nm} \mathrm{~min}{ }^{-1}$. UV-visible absorption studies were performed on a Shimadzu UV-2101 PC. One ml solutions were used for collecting the spectrum using a faster scan speed and slit width of $5 \mathrm{~nm}$ in the range of 200-800 nm. Far-UV CD spectra were collected using a Jasco J-815 CD spectrometer. The path length of the cuvette was $0.1 \mathrm{~cm}$ and the bandwidth of the light used was $1 \mathrm{~nm}$. Three scans were recorded with a scan speed of $100 \mathrm{~nm} \min ^{-1}$ and the scans were averaged.

\section{Spectroscopy and microscopy studies}

The matrix assisted laser desorption ionization (MALDI) studies were performed on AutoflexIII TOF/TOF (MALDI-TOF mass spectrometer, Bruker Daltonics Co.) using sinapinic acid as a matrix and by using a $355 \mathrm{~nm}$ laser as an ionization source. The FTIR studies were performed using a PerkinElmer FT-IR spectrometer. One mg of lyophilised sample of lyz-Cu NCs was ground with $\mathrm{KBr}$ and a pellet was made from this. The spectrum for only lysozyme was also recorded for comparison. In order to carry out field emission gun transmission electron microscopy (FEG-TEM) or just TEM microscopy studies, samples were spread over carbon coated copper grids having 200 mesh and dried and analyzed by using JEOL JEM-2100F (FEG-TEM) operating at $200 \mathrm{kV}$. X-ray photoelectron spectroscopy (XPS) was performed using $\mathrm{Mg} \mathrm{K} \alpha$ excitation $(1253.6 \mathrm{eV}$ ) with a VG ESCALAB MKII spectrometer. Calibration for the binding energy was done based on carbon $1 \mathrm{~s}$ being at $284.6 \mathrm{eV}$.

\section{Results and discussion}

\section{Lysozyme coated copper nanoclusters (lyz-CuNCs)}

The lyz-CuNCs were initially prepared from a solution of lysozyme and copper(II) chloride at $\mathrm{pH}=12$ upon treating with a mild reducing agent, such as hydrazine, and the solution was adjusted to the requisite $\mathrm{pH}$ using $\mathrm{HCl}$. The lyz-CuNCs were characterized by UV-Vis absorption, fluorescence, CD and XPS spectroscopy, and also by TEM.

\section{Emission and absorption studies}

Among the studies carried out at $\mathrm{pH}=12,10,7,5$ and 3, the one that is studied at the neutral $\mathrm{pH}$, i.e., a mixture of lyz and copper salt solution (details given in the experimental section) resulted in dark yellow colour after one day of incubation and the fluorescence emission suggested the formation of CuNCs. Photographs of the vials of lyz-CuNCs in aqueous solution at all $\mathrm{pH}$ values under visible and UV light are given in Fig. 1 . At $\mathrm{pH}=$ 12 and 10, the solutions were transparent and exhibited no green fluorescence when observed under UV light. The green fluorescence was stronger at neutral $\mathrm{pH}$. However, there was only a minimal amount of green fluorescence at $\mathrm{pH}=5$ and

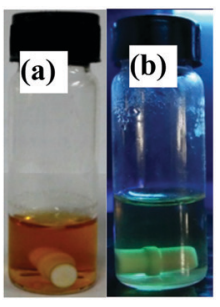

pH

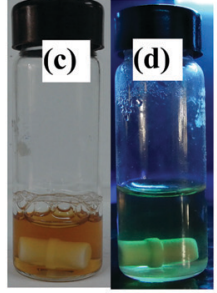

10

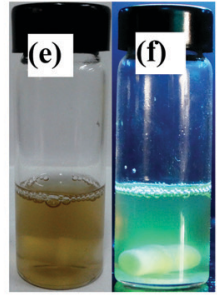

7

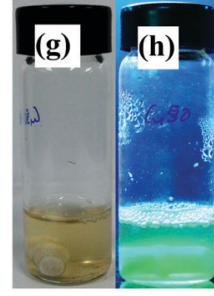

5

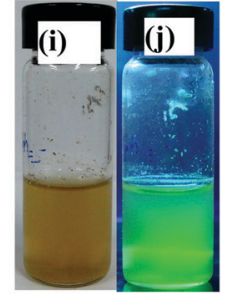

3

Fig. 1 Photographs of lyz-CuNCs in aqueous solution under visible (left side vial) and UV (right side vial) light in each pair at the indicated pH. 
3 due to the precipitation and/or agglomeration. In the literature, lysozyme capped CuNCs were synthesized at $\mathrm{pH}$ 11-12 and a temperature of $40-45{ }^{\circ} \mathrm{C}$, but using two different ratios of protein to the copper salt to result in blue emission $\left(\lambda_{\mathrm{ex}}=\right.$ $\left.360 \& \lambda_{\mathrm{em}}=450 \mathrm{~nm}\right)$ in one case ${ }^{13}$ and red emission $\left(\lambda_{\mathrm{ex}}=365 \&\right.$ $\lambda_{\mathrm{em}}=600 \mathrm{~nm}$ ) in the other. ${ }^{14}$ Therefore, the literature reports of lyz-CuNCs sharply differ from our case where our lyz-CuNCs were synthesized at neutral $\mathrm{pH}$ and RT and these show green fluorescence with $\lambda_{\mathrm{em}}=510 \mathrm{~nm}$, and not the blue or the red emission. While the excitation is in the UV region (360-365 nm), in the present case the excitation is in the visible region at $490 \mathrm{~nm}$. Thus the lyz-CuNCs reported in this paper exhibited a shift of $60 \mathrm{~nm}$ in their fluorescence emission when compared to the blue lyz-CuNCs reported in the literature. ${ }^{13}$ Thus, our green fluorescent lyz-CuNCs complement the blue ${ }^{13}$ and red $^{14}$ nanoclusters reported in the literature by completing the range of emission of 450 to $600 \mathrm{~nm}$ via bridging the gap with its emission centered at $510 \mathrm{~nm}$.

As compared to other $\mathrm{pH}$ conditions studied, at $\mathrm{pH}=7$, the agglomeration is prevented due to the capping of lyz that results in exhibiting significant fluorescence emission intensity at $510 \mathrm{~nm}$ (Fig. 2a). Minimal fluorescence emission intensity was noticed for the solution of lyz-CuNCs at $\mathrm{pH}=5$ and no fluorescence intensity was noticed for that studied at $\mathrm{pH}=12$, 10 and 3. Solution of lyz-CuNCs was dialyzed for removing unbound copper ions as well as other small molecular components. Even after the dialysis, the solutions exhibited fluorescence emission for the study carried out at $\mathrm{pH}=7$ (Fig. 2b). The quantum yields of the lyz-CuNCs determined as given in the experimental section are $0.014,0.016,0.18,0.037$ and 0.0093 at $\mathrm{pH}$ values of $3,5,7,10$ and 12 respectively, with respect to quinine hemisulfate, thus showing the highest quantum yield at $\mathrm{pH}=7$. The protein lyz alone shows maximum emission at $\sim 350 \mathrm{~nm}$ upon excitation at $280 \mathrm{~nm}$ at all the $\mathrm{pH}$ values studied due to the presence of aromatic amino acids, viz., Trp and Tyr as can be noticed from Fig. 2c. When excited at $490 \mathrm{~nm}$, lyz alone doesn't show any fluorescence (Fig. 2d) which suggested that the green fluorescence is emitted only by the protein coated CuNPs, viz., lyz-CuNCs, but not simply by the protein.
The absorption spectrum of lysozyme alone shows a typical protein absorption spectrum with a maximum peak at $280 \mathrm{~nm}$ and without any SPR band at all the $\mathrm{pH}$ studied (Fig. 3a). However, the lyz-CuNCs show two peaks, one broad peak at $\sim 300 \mathrm{~nm}$ and a sharp peak at $\sim 220 \mathrm{~nm}$ (Fig. 3b). In the absorption spectra at $\mathrm{pH}=7$, no $\mathrm{SPR}$ band was noticed at $550 \mathrm{~nm}$ due to smaller size of the copper nanoparticles as measured based on TEM (3-5 $\mathrm{nm})$ and these are referred to as nanoclusters (NCs) (Fig. 3b and c). Thus we find a significant increase in the absorbance of the SPR band observed at $\sim 550 \mathrm{~nm}$ in the case of $\mathrm{pH}=12$ which distinguishes this from the remaining $\mathrm{pH}$ values and this supports the nanoparticle formation as can be noted from the inset of Fig. 3b. Thus, the SPR band almost diminishes as the $\mathrm{pH}$ of the solution decreases and turns to acidic (Fig. 3b). The spectrum of lyz alone is totally different from the absorption spectra obtained for lyz-CuNCs and the latter is characteristic of the copper bound lyz (Fig. 3a and b).

\section{CD and XPS studies}

While the simple lyz is predominantly present in the $\alpha$-helical (222 $\mathrm{nm}$ band in CD) structure, the lyz-CuNCs exhibit mostly a random coil ( $200 \mathrm{~nm}$ band in CD) like structure under all the $\mathrm{pH}$ conditions studied (Fig. 4a). Even after the dialysis, at $\mathrm{pH}=7$, the random coil structure was still seen to be predominant (Fig. 4b). This suggested the partial denaturation of the protein in lyz-CuNCs which helps in opening an $\alpha$-helix as well as in reducing $\mathrm{Cu}^{2+}$ to $\mathrm{Cu}^{0}$ and the protein, lyz is coated onto the CuNCs. XPS analysis was performed with lyz-CuNCs obtained after the dialysis. The XPS spectra were characteristic of the reduction of $\mathrm{Cu}^{2+}$ to $\mathrm{Cu}^{0}$ both based on the main peak as well as the satellite peaks. While the $\mathrm{Cu}^{2+}$ shows two strong satellites of which one is at $962 \mathrm{eV}$, the $\mathrm{Cu}^{0}$ shows two weak satellites with the main peak being centered around $933 \mathrm{eV}$. All these features can be clearly seen from the XPS spectra given in Fig. $4 \mathrm{c}$ and the spectra correspond to the $\mathrm{Cu}^{0}$ species.

\section{Characterization by MALDI and FTIR studies}

The lysozyme, in its native state, showed a $\mathrm{m} / \mathrm{z}$ peak around $14300 \mathrm{Da}$ (for $z=1$ ) and $7145 \mathrm{Da}$ (for $z=2$ ). Upon addition of
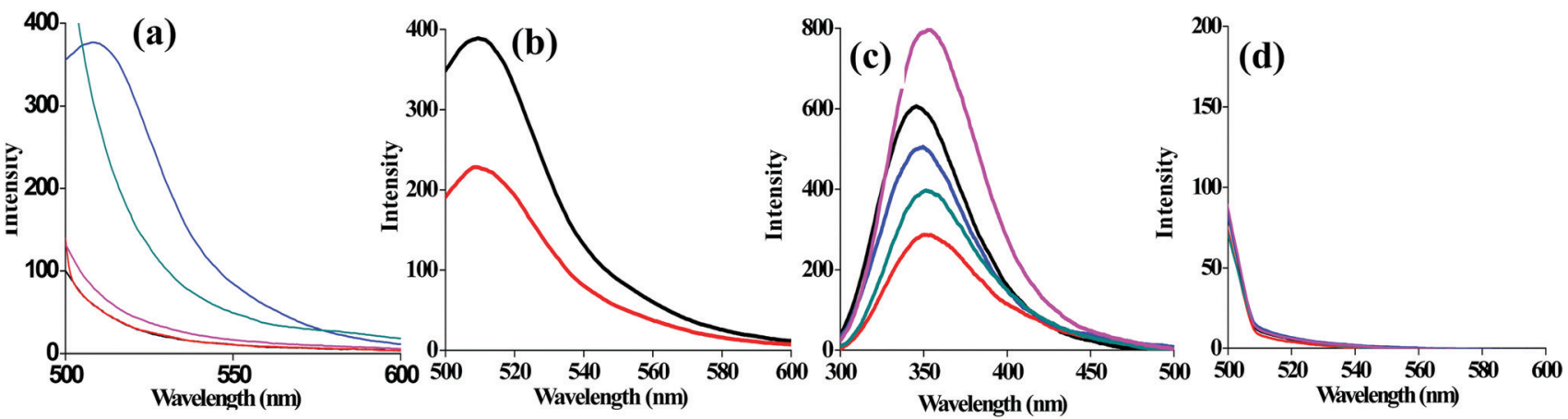

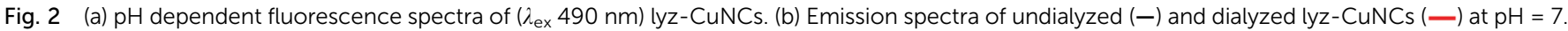

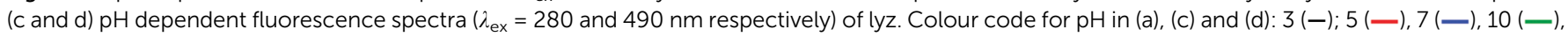
12 (-). 

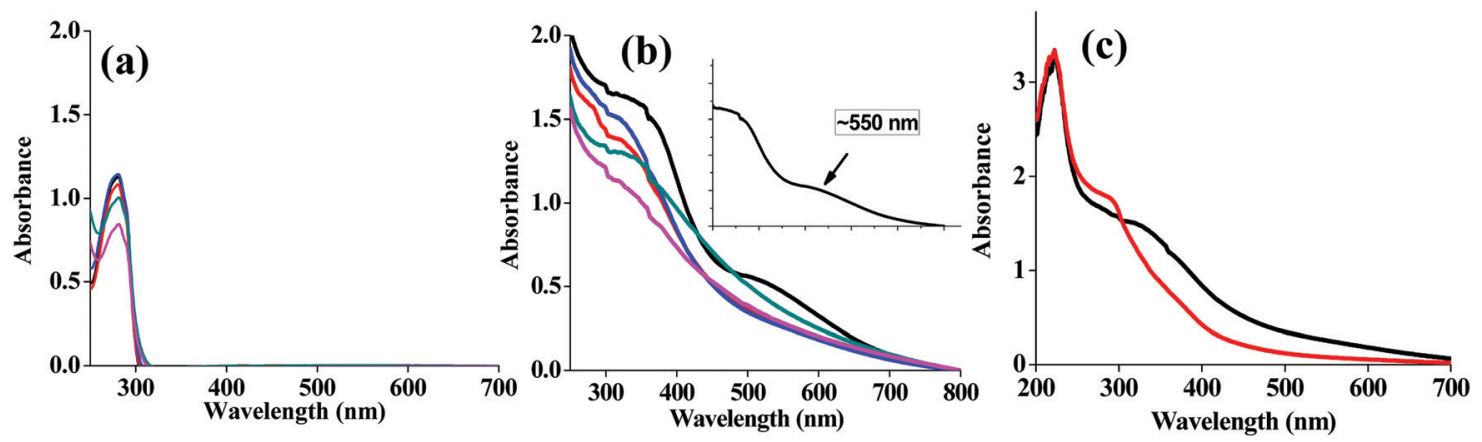

Fig. $3 \mathrm{pH}$ dependent absorption spectra of (a) lysozyme alone and (b) lyz-CuNPs. The SPR band is shown in the inset. The colour code for pH in both (a) and (b) is: $12(-) ; 10(-), 7$ (-), 5 (-), 3 (-). (c) Absorption spectra of undialyzed (-) and dialyzed lyz-CuNCs (-) at pH = 7.
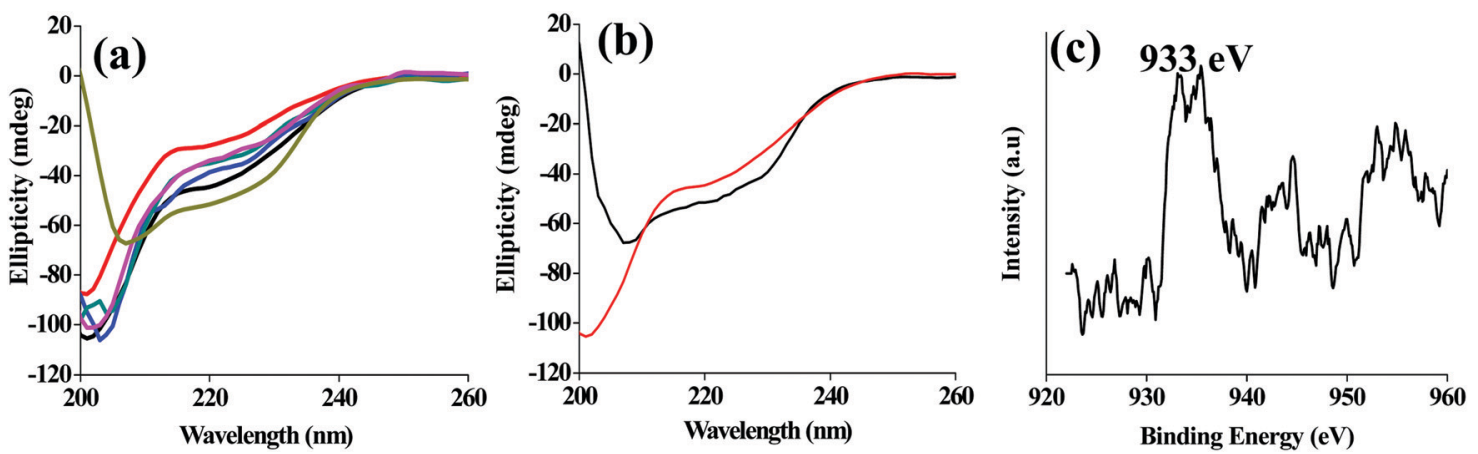

Fig. 4 (a) Circular dichroism spectra of lyz-CuNPs at different pH values: $12(-), 10$ (-), 7 (-), 5 (-), 3 (—), and simple lyz at pH = 7 (-). (b) CD spectra of simple lyz (-) and dialyzed lyz-CuNCs $(-)$ at $\mathrm{pH}=7$. (c) The XPS spectrum of copper in lyz-CuNCs (dialyzed) at pH $=7$.

20 equivalents of $\mathrm{Cu}^{2+}$ at $\mathrm{pH} 10$, the peak shifts in the molecular weight corresponding to the presence of higher copper clusters, such as $\mathrm{Cu}_{1}, \mathrm{Cu}_{2}, \mathrm{Cu}_{4}$ and $\mathrm{Cu}_{6}$ as noticed from the $m / 2$ peak (Fig. 5a). Under these conditions, the peak corresponding to the protein alone almost completely diminished and the copper bound ones were noticed as marked in the spectra. Upon addition of hydrazine, the $\mathrm{Cu}^{2+}$ ion is reduced to $\mathrm{Cu}^{0}$ and results in a lysozyme bound nanocluster which was then dialyzed. As the desorption of the dialysed nanocluster is not abundant, the corresponding MALDI spectra resulted in greater noise. In the case of blue fluorescent lyz-CuNCs, 2 to 9 copper bound nanoclusters $\left(\mathrm{Cu}_{2}, \mathrm{Cu}_{4}\right.$ and $\mathrm{Cu}_{9}$ ) were reported, ${ }^{13}$ while no MALDI studies were reported in the case of red lyz-CuNCs. ${ }^{14}$ An FTIR spectrum of simple protein shows an amide peak at $\sim 1600 \mathrm{~cm}^{-1}$ along with a shoulder at $\sim 1700 \mathrm{~cm}^{-1}$. In the case of lyz-CuNCs, only the shoulder disappears and the amide band is retained, suggesting the capping of CuNCs by the protein, lysozyme. Thus, the protein acts as a capping as well as a stabilizing agent in these CuNCs (Fig. 5b).
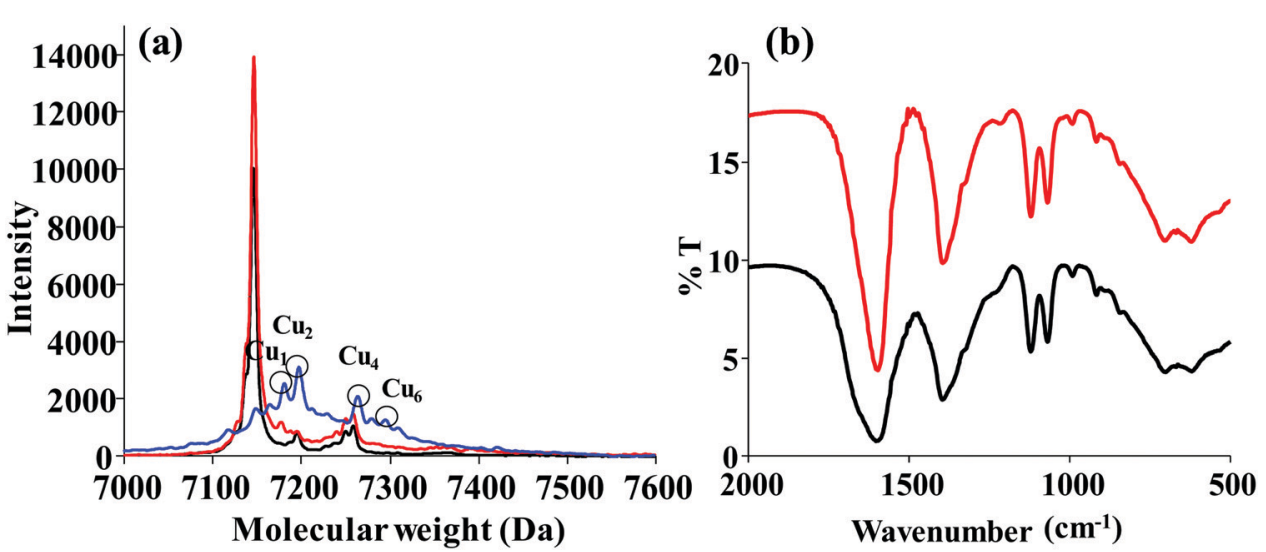

Fig. 5 (a) MALDI-TOF spectra of simple lyz (-) and the lyz treated with $\mathrm{Cu}^{2+}$ in a $1: 20$ ratio at pH 7 ( -) and at pH 10 ( -). (b) FTIR spectra of simple lyz $(-)$ and lyz-CuNCs (-). 


\section{TEM studies}

The TEM micrographs were measured for lyz-CuNCs at $\mathrm{pH}=7$ both before and after the dialysis. Before the dialysis, the lyzCuNCs exhibited particle sizes in the range of 5-20 nm (Fig. 6a, e and $\mathrm{f}$ ). The protein coating in lyz-CuNCs was proven by EDAX, which shows peaks for copper along with N, C, O, and S (Fig. 6b) and particle size distribution is shown in the inset. Since some larger size particle of 10-20 nm was noticed in this sample, TEM was performed again with the dialyzed and centrifuged solution for establishing the size distribution of lyz-CuNCs. After the dialysis, the particle size distribution was restricted to $3-5 \mathrm{~nm}$ (Fig. $6 \mathrm{c}, \mathrm{d}$, g and $\mathrm{h}$ ). The particles in the size distribution (Fig. $6 \mathrm{~d}$ ) of 3-5 $\mathrm{nm}$ of lyz-CuNCs were expected to show fluorescence emission and these indeed showed green fluorescence as presented in this paper.

At acidic $\mathrm{pH}$, the size of lyz-CuNCs was in the range of 5-10 $\mathrm{nm}$ but these nanoparticles were precipitated out from the solution and hence weak fluorescence was noticed (Fig. 6i, j and $7 \mathrm{a}, \mathrm{b})$. The micrograph indicates a clear-cut aggregation of the small nanoparticles into clusters of 50-100 nm size. Even the EDAX measurement (Fig. 61 and 7d) carried out with the samples at $\mathrm{pH}=5$ and 3 clearly showed the protein coating by exhibiting the peaks for $\mathrm{C}, \mathrm{N}, \mathrm{O}$, and $\mathrm{S}$ along with copper.
On going from $\mathrm{pH}=5$ to 3 , spherical aggregates are more prevalent. Thus, TEM clearly supports the fact that 3-5 $\mathrm{nm}$ size lyz-CuNCs are formed mainly at $\mathrm{pH}=7$ and these give rise to green emission, while the particles observed at all other $\mathrm{pH}$ values are of larger size or aggregate leading to minimal or no fluorescence. At $\mathrm{pH}=5$ and 3, the HR-TEM image shows an interplanar distance of 0.21 and $0.19 \mathrm{~nm}$ respectively that corresponds to the (111) lattice planes of metallic copper, supporting the reduction of $\mathrm{Cu}^{2+}$ to $\mathrm{Cu}^{0}$ (Fig. $6 \mathrm{k}$ and $7 \mathrm{c}$ ). The presence of $\mathrm{Cu}^{0}$ was already shown by XPS. The interplanar distance observed in the present case agrees well with that reported in the literature. ${ }^{25}$ At $\mathrm{pH}=12$ and 10, larger aggregates of the small nanoclusters were noticed clearly showing the agglomeration at these $\mathrm{pH}$ values to result in fiber like structures (Fig. 7e-h).

\section{Cell viability of the fluorescent lyz-CuNCs prepared at $\mathbf{p H}=7$}

The MTT assay was carried out to determine the cell viability of lyz-CuNCs upon treating the NIH3T3 cells with these nanoclusters and incubating these for a period of $24 \mathrm{~h}$. The cell viability was studied as a function of the concentration of lyz-CuNCs as shown in Fig. 8. At up to $20 \mu \mathrm{g} \mathrm{ml}^{-1}$ concentration of lyz-CuNCs, the cell viability is $\sim 100 \%$. Even at very high concentration of lyz-CuNCs
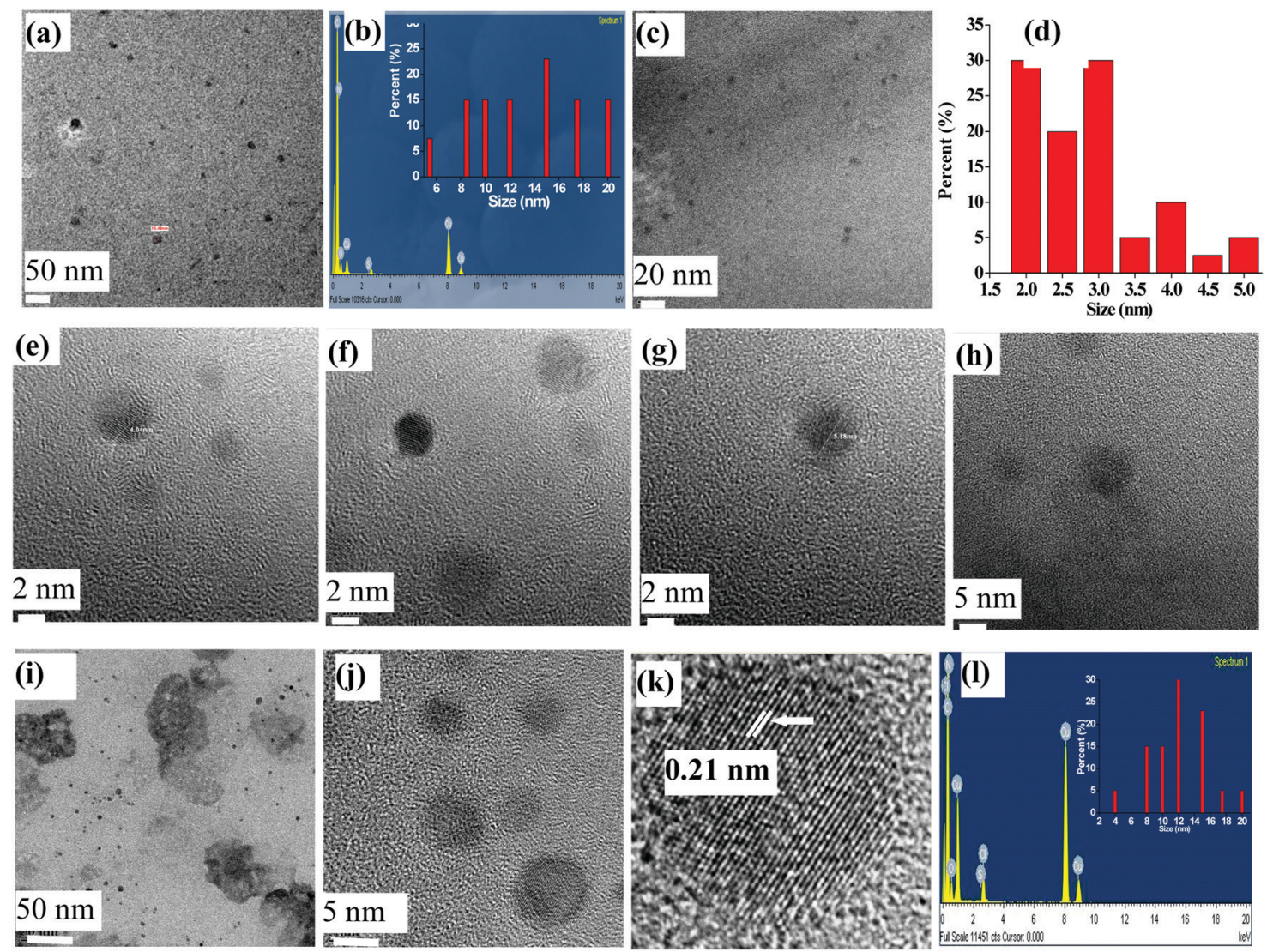

Fig. 6 lyz-CuNCs at pH = 7. (a) TEM image before dialysis (scale bar is $50 \mathrm{~nm}$ ). (b) EDAX of the sample before dialysis. The inset bar diagram is a particle size distribution plot. (c) TEM image after dialysis (scale bar is $20 \mathrm{~nm}$ ). (d) Particle size distribution after dialysis. HR-TEM of lyz-CuNCs at pH 7.0: (e and f) before and ( $\mathrm{g}$ and $\mathrm{h}$ ) after dialysis. ( $\mathrm{i}$ and j) TEM and ( $\mathrm{k}$ ) HR-TEM images of lyz-CuNCs at pH = 5. The scale bar in (i) is $50 \mathrm{~nm}$ and in (j) is $5 \mathrm{~nm}$. (l) EDAX of the lyz-CuNCs at $\mathrm{pH}=5$. The inset in (l) is the particle size distribution plot wherein the $x$-axis corresponds to size (nm) and the $y$-axis corresponds to percent (\%) of the nanoparticles. 

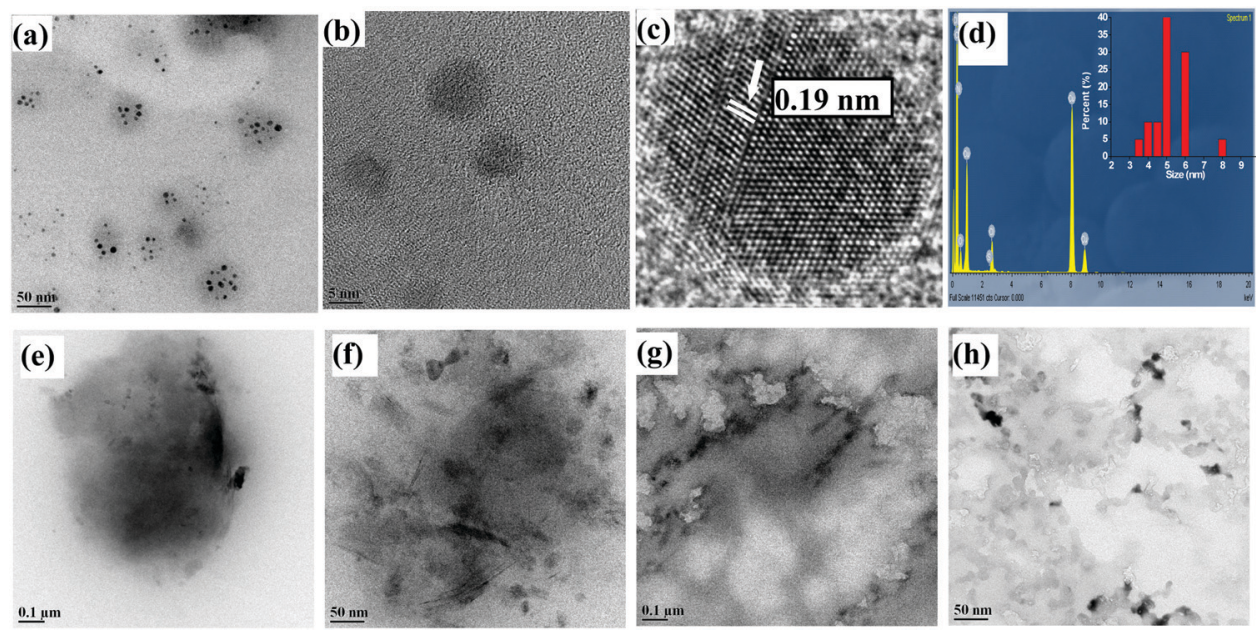

Fig. 7 (a and b) TEM and (c) HR-TEM images of the lyz-CuNCs at pH = 3. The scale bar in (a) is $50 \mathrm{~nm}$ and in (b) is $5 \mathrm{~nm}$. (d) EDAX of the same. The inset in (d) is the particle size distribution plot wherein the $x$-axis corresponds to size $(\mathrm{nm})$ and the $y$-axis corresponds to percent (\%) of the nanoparticles. TEM images of the lyz-CuNPs at $\mathrm{pH}=12$ (e and f) and at pH = 10 ( $\mathrm{g}$ and h). The scale bars are 100, 50, 100 and $50 \mathrm{~nm}$ respectively in (e), (f), (g) and (h).

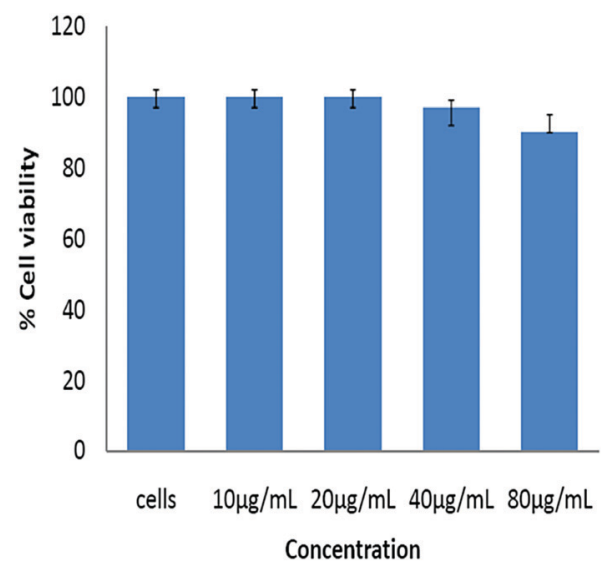

Fig. 8 Percent viability of NIH3T3 cells after the treatment with various concentrations of lyz-CuNCs.

(i.e., $80 \mu \mathrm{g} \mathrm{ml} \mathrm{m}^{-1}$ ), $\sim 90 \%$ cell viability was noticed, supporting that these nanoclusters are non-toxic to cells and hence are well suited for cellular imaging work.

\section{Cell imaging by the fluorescent lyz-CuNCs prepared at $\mathbf{p H}=7$}

The utility of lyz-CuNCs in cellular imaging was successfully demonstrated using three cell lines, namely, NIH3T3 cells (mouse embryonic fibroblast cell), MCF7 cells (human breast cancer cells) and MDA-MB-231 cells (human estrogen negative breast cancer cells) using fluorescence confocal microscopy. The fluorescence image collected under $490 \mathrm{~nm}$ excitation showed bright green fluorescence in cells (Fig. 9). In all the three types of cells, green fluorescence was mostly located in the periphery, though some fluorescence intensity was seen inside the cells particularly in the case of MCF7 cells.

\section{Evidence for the cellular uptake of lyz-CuNCs}

Initially, the cellular uptake of lyz-CuNCs was studied using the same three cell lines, viz., NIH3T3, MCF7 and MDA-MB-231 using fluorescence confocal microscopy. The localization of lyz-CuNCs was determined in L929 cells by the Z-stack experiment and it is noted that lyz-CuNCs were present in the cells predominantly in the perinuclear cytoplasm (Fig. 10). The fluorescence in blue lines in $Y Z$ and $X Z$ axes clearly indicate that lyz-CuNCs entered the cells and mostly localized in the cytoplasm.

\section{Conclusions and comparisons}

Lysozyme was chosen as the model protein to stabilize the copper nanoclusters to give lyz-CuNCs. In the literature, lyz was used for the synthesis of gold nanoparticles within a single crystal of lysozyme. ${ }^{26,27}$ In our paper, we have shown that the protein, lyz, stabilizes the copper nanoparticles due to its high $\mathrm{p} K_{\mathrm{a}}$ over a wide $\mathrm{pH}$ range. Lysozyme is a monomer containing 129 amino acid residues with 4 disulfide bridges and has a molecular weight of $14.3 \mathrm{kDa}^{26}$ The presence of $14 \%$ basic amino acids, such as Lys and Arg, makes this protein well suited for binding to copper depending on the $\mathrm{pH}$ and this has been clearly demonstrated from the studies reported in this paper by carrying out these at different $\mathrm{pH}$ and varying protein to copper mole ratios.

The fluorescence intensity of lyz-CuNCs observed at $\mathrm{pH}=7$ was high with a quantum yield of 0.18 with respect to quinine hemisulfate. The small size (3-5 nm) of lyz-CuNCs results in the formation of fluorescent nanoclusters. As compared to other $\mathrm{pH}$ values studied, at $\mathrm{pH}=7$, the TEM image showed relatively homogeneous distribution of 3-5 $\mathrm{nm}$ particles without any significant aggregation of proteins resulting in green fluorescence. However, at both lower and higher $\mathrm{pH}$, aggregated species of different types were observed in TEM, supporting that at neutral $\mathrm{pH}$ the binding of copper dominates resulting in smaller size nanoclusters to give green fluorescence emission. A schematic representation of the nanoparticle formation under different $\mathrm{pH}$ conditions is shown in Fig. 11a-c.

To our knowledge, this is the first example of the formation of protein mediated green fluorescent copper nanoparticles. 

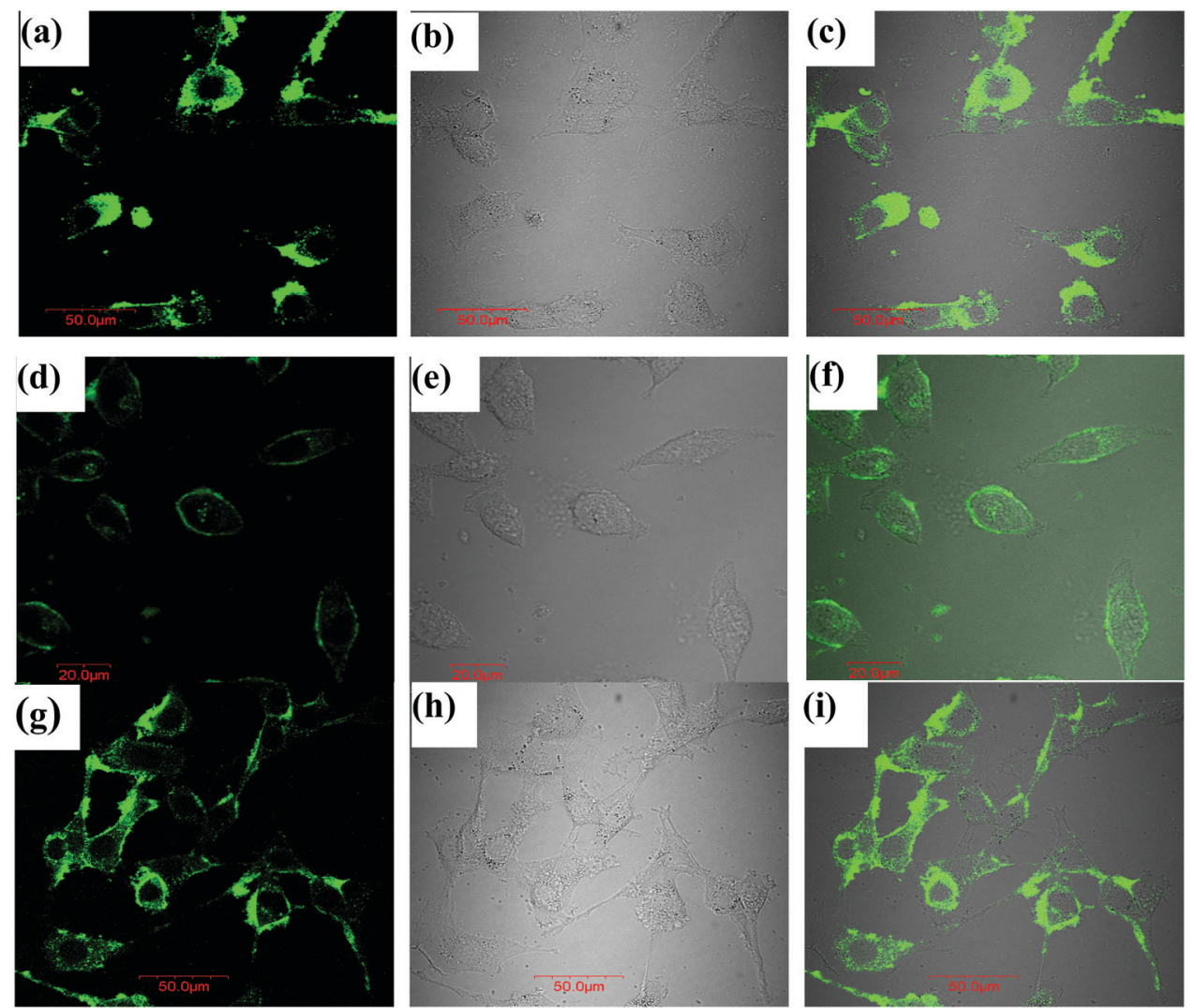

Fig. 9 Confocal microscopy images of the cells incubated with $10 \mu \mathrm{g} \mathrm{ml}^{-1}$ lyz-CuNCs for $12 \mathrm{~h}$ : (a-c) fluorescent, bright field and overlay of images from NIH3T3 normal cells (mouse embryonic fibroblast cells). (d-f) The same type but from MCF7 cells (human breast cancer cells). (g-i) The same type but from MDA MB 231 cells (human estrogen negative breast cancer cells).

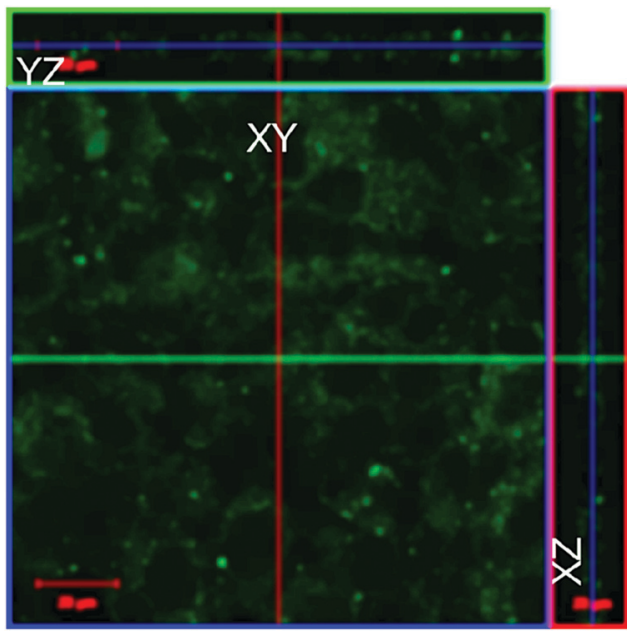

Fig. 10 Ortho-view images of Z-stack of L292 cells showing lyz-CuNCs in the cells.

Blue and red fluorescent CuNCs stabilized with protein, DNA and ascorbic acid were reported in the literature. ${ }^{12-18}$ However, in the present study, the emission is around $\sim 510 \mathrm{~nm}$ owing to its green fluorescence. The green fluorescence reported in our paper emerges from the clusters of size 3-5 $\mathrm{nm}$ when the study was carried out at neutral $\mathrm{pH}$.
The report of our green fluorescent lyz-CuNCs complements the blue $\left(\lambda_{\mathrm{ex}}=360 \& \lambda_{\mathrm{em}}=450 \mathrm{~nm}\right)^{13}$ and red $\left(\lambda_{\mathrm{ex}}=365 \& \lambda_{\mathrm{em}}=\right.$ $600 \mathrm{~nm})^{14}$ nanoclusters reported in the literature by completing the range of emission of 450 to $600 \mathrm{~nm}$ through bridging the gap with its emission centered at $510 \mathrm{~nm}$. The green fluorescence property of the lyz-CuNCs has been proven to be useful in the cell imaging of normal (NIH3T3 cells) as well as cancerous cells (MCF7 and MDA-MB-231 cells). As reported in the literature, the lyz-CuNCs were used for imaging only the HeLa cell lines. ${ }^{13}$ Our study reports the imaging of one healthy cell line (NIH3T3 cells) and two cancerous cells (MCF7 and MDA-MB-231 cells) along with proving its presence inside the cell by Z-stacking experiment. The Z-stacking experiment showed that lyz-CuNCs go inside the cell and are localized in the cytoplasm. Thus, our lyz-CuNCs provide broader applicability for cell or tissue imaging. Our attention was attracted towards green fluorescent CuNPs due to their similarity to the green fluorescent protein (GFP) reported in the literature. ${ }^{25}$ Similar to the present lyz-CuNCs, the GFP from A. victoria has its excitation peak at $395 \mathrm{~nm}$ and the emission peak at $509 \mathrm{~nm} .{ }^{28}$ GFP is widely used for cell labeling and as a marker for gene expression. ${ }^{29-31}$ The lyz-CuNC material can also be used in labeling various types of cells similar to that where GFP is being used. Comparison of these two in the case of cancer cell labeling can be viewed from Fig. 11d and e. In our case the 
(a) $\mathrm{pH}=12$ and 10

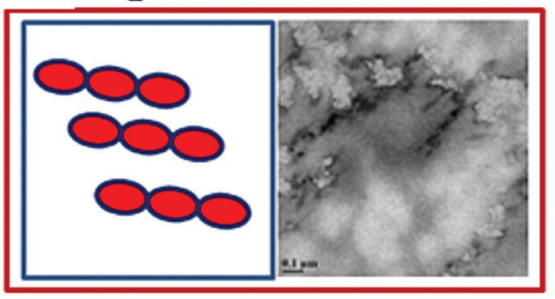

lyz-CuNPs aggregates

(c) pH 5 and 3
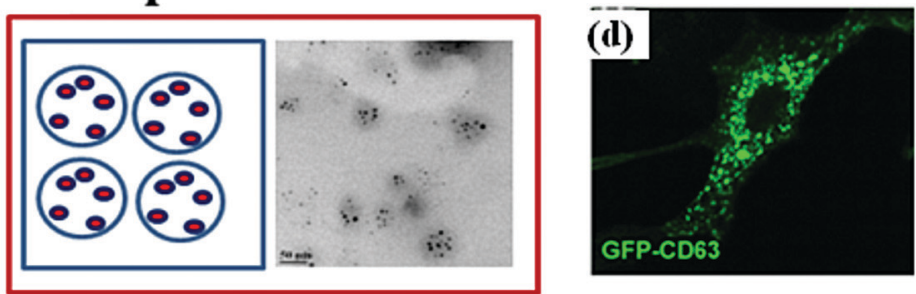

(b) $\mathbf{p H}=\mathbf{7}$

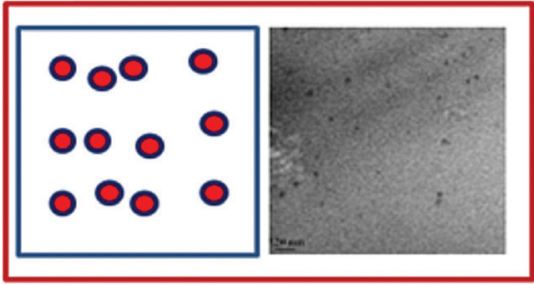

lyz-CuNCs (3-5 nm)

\section{Aggregates of lyz-CuNCs}

Fig. $11(\mathrm{a}-\mathrm{c})$ The schematic representation of the formation of lyz-CuNPs and lyz-CuNCs under the indicated pH conditions. Confocal microscopy images: (d) BT-CD63 cells labeled with GFP-CD63 exosomes ${ }^{30}$ and (e) MCF7 cells labeled with lyz-CuNCs at pH 7.

excitation wavelength is clearly in the visible region unlike those reported in the literature regarding the blue and the red copper nanoclusters wherein the excitation wavelength was in the UV region. BT-CD63 cells labeled with GFP-CD63 exosomes exhibit bright green fluorescence. ${ }^{30}$ Similar green fluorescence was also noticed in our studies of lyz-CuNCs with MCF-7 cells. Hence, green fluorescent lyz-CuNCs can be used for cancer diagnosis and treatment when appropriately tuned and conjugated with a drug molecule. Therefore, the green fluorescent lyz-CuNCs can be an alternate choice for GFP, since the GFP needs a tedious procedure to express, purify and conjugate and is also expensive to procure. Hence these lysozyme coated copper nanocluster (lyz-CuNC) materials may find real time applications in biology, medical diagnosis and even in therapy when appropriately tuned.

\section{Conflicts of interest}

The authors declare no competing financial interest.

\section{Acknowledgements}

CPR acknowledges the Department of Science and Technology for the JC Bose National Fellowship (SB/S2/JCB/066/2015) and IIT Tirupati for providing an MHRD Professorship. CPR expresses his gratitude to IIT Bombay for fostering his academic and research activities for over three decades. We thank the central facilities, such as TEM, XPS, MALDI-TOF and confocal microscopy of IIT Bombay, for providing services.

\section{References}

1 B. S. González, M. J. Rodríguez, C. Blanco, J. Rivas, M. A. L'opez-Quintela and J. M. G. Martinho, Nano Lett., 2010, 10, 4217-4221.

2 S. I. Tanaka, J. Miyazaki, D. K. Tiwari, T. Jin and Y. Inouye, Angew. Chem., Int. Ed., 2011, 50, 431-435.

3 C. C. Huang, Z. Yang, K. H. Lee and H. T. Chang, Angew. Chem., Int. Ed., 2007, 46, 6824-6828.

4 M. Hyotanishi, Y. Isomura, H. Yamamoto, H. Kawasaki and Y. Obora, Chem. Commun., 2011, 47, 5750-5752.

5 G. P. Jose, S. Santra, S. K. Mandal and T. K. Sengupta, J. Nanobiotechnol., 2011, 9, 9-15.

6 H. Zhang, T. Watanabe, M. Okumura, M. Haruta and N. Toshima, Nat. Mater., 2012, 11, 49-52.

7 Z. Wu, M. Wang, J. Yang, X. Zheng, W. Cai, G. Meng, H. Qian, H. Wang and R. Jin, Small, 2012, 8, 2028-2035.

8 W. J. Cho, Y. Kim and J. K. Kim, ACS Nano, 2012, 6, 249-255.

9 N. Vilar-Vidal, M. C. Blanco, M. A. Lopez-Quintela, J. Rivas and C. Serra, J. Phys. Chem. C, 2010, 114, 15924-15930.

10 H. Kawasaki, Y. Kosaka, Y. Myoujin, T. Narushima, T. Yonezawa and R. Arakawa, Chem. Commun., 2011, 47, 7740-7742.

11 W. Wei, Y. Lu, W. Chen and S. Chen, J. Am. Chem. Soc., 2011, 133, 2060-2063.

12 N. Goswami, A. Giri, M. S. Bootharaju, P. L. Xavier, T. Pradeep and S. K. Pal, Anal. Chem., 2011, 83, 9676-9680.

13 R. Ghosh, A. K. Sahoo, S. S. Ghosh, A. Paul and A. Chattopadhyay, ACS Appl. Mater. Interfaces, 2014, 6, 3822-3828. 
14 C. Wang, S. Shu, Y. Yao and Q. Song, $R S C A d v ., 2015,5$, 101599-101606.

15 Y. Guo, F. Cao, X. Lei, L. Mang, S. Cheng and J. Song, Nanoscale, 2016, 8, 4852-4863.

16 S. Kim, J. H. Kim, W. Y. Kwon, S. H. Hwang, B. S. Cha, J. M. Kim, S. S. Oh and K. S. Park, Microchim. Acta, 2019, 186, 479-484.

17 S. Momeni, R. Ahmadi, A. Safavi and I. Nabipour, Talanta, 2017, 175, 514-521.

18 C. Wang, C. Wang, L. Xu, H. Cheng, Q. Lin and C. Zhang, Nanoscale, 2014, 6, 1775-1781.

19 H. Kawasaki, K. Hamaguchi, I. Osaka and R. Arakawa, Adv. Funct. Mater., 2011, 21, 3508-3515.

20 H. Schwalbe, S. B. Grimshaw, A. Spencer, M. Buck, J. Boyd, C. M. Dobson, C. Redfield and L. J. Smith, Protein Sci., 2001, 10, 677-688.

21 S. Chandirasekar, J. G. You, J. H. Xue and W. L. Tseng, J. Mater. Chem. B, 2019, 10, 3876-3883.
22 B. A. Russell, B. Jachimska, P. Komorek, P. A. Mulheran and Y. Chen, Phys. Chem. Chem. Phys., 2017, 19, 7228-7235.

23 S. Pang and S. Liu, Anal. Methods, 2017, 9, 6713-6718.

24 X. Zhou, R. Lai, H. Li and C. I. Stains, Anal. Chem., 2015, 87, 4081-4086.

25 A. Chahadih, H. E. Hamzaoui, O. Cristini, L. Bigot, R. Bernard, C. Kinowski, M. Bouazaoui and B. Capoen, Nanoscale Res. Lett., 2012, 7, 1-6.

26 M. Liang, L. Wang, X. Liu, W. Qi, R. Su, R. Huang, Y. Yu and Z. He, Nanotechnology, 2013, 24, 245601.

27 H. Wei and Y. Lu, Chem. - Asian J., 2012, 7, 680-683.

28 M. Ormö, A. B. Cubitt, K. Kallio, L. A. Gross, R. Y. Tsien and S. J. Remington, Science, 1996, 273, 1392-1395.

29 M. Chalfie, Y. Tu, G. Euskirchen, W. W. Ward and D. C. Prasher, Science, 1994, 263, 802-805.

30 R. B. Koumangoye, A. M. Sakwe, J. S. Goodwin, T. Patel and J. Ochieng, PLoS One, 2011, 6, e24234.

31 M. H. Robert, Lab. Invest., 2015, 95, 432-452. 